
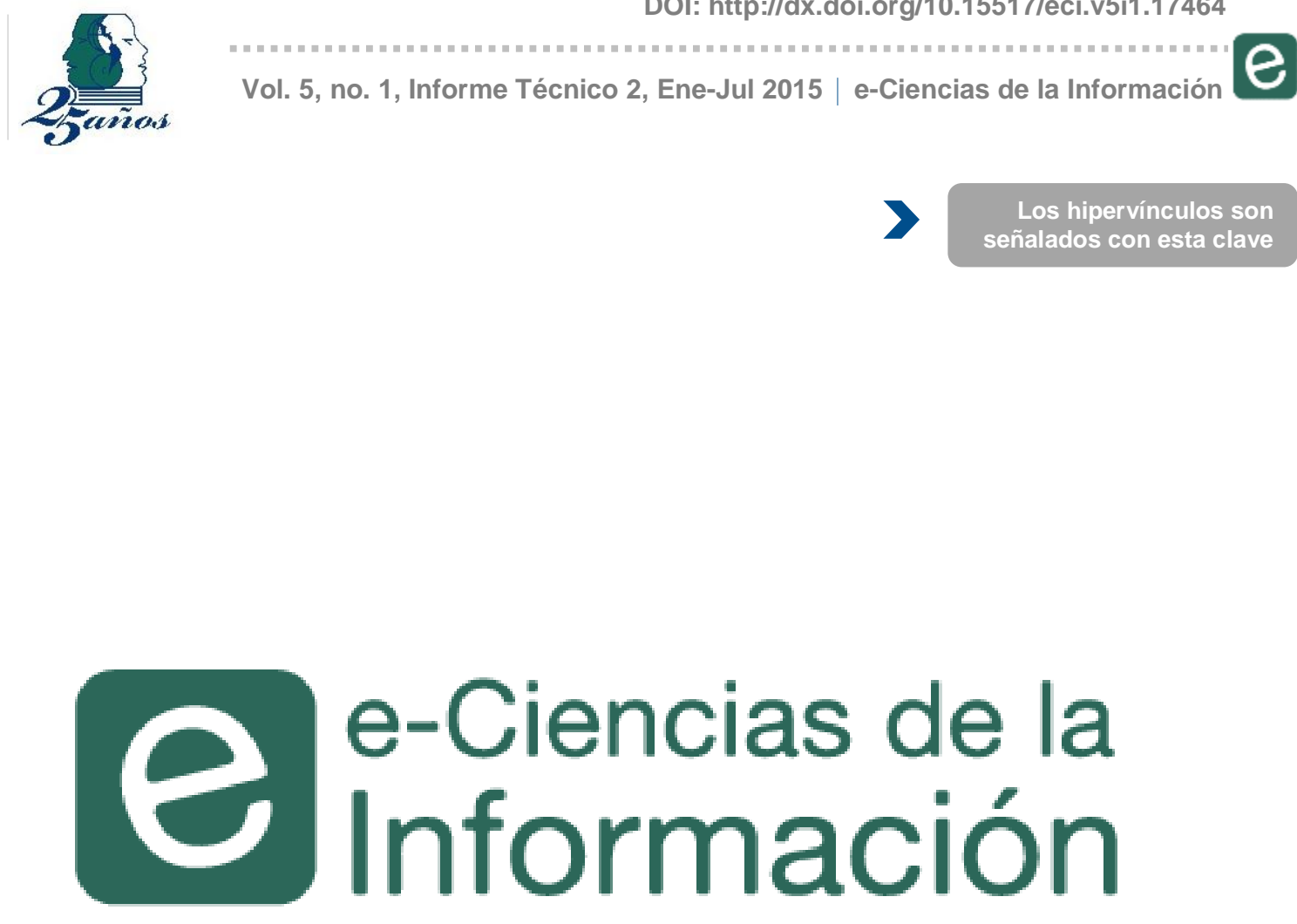

\title{
$>$ Consolidación del Posgrado en Bibliotecología de la Universidad de Costa Rica
}

$>$ Ruth González-Arrieta Publicado 01 de enero, 2015 / Informe Técnico 2

\section{()ㅜ잉}

Universidad de Costa Rica

Visite el sitio web de e-Ciencias de la Información 


\title{
Consolidación del Posgrado en Bibliotecología de la Universidad de Costa Rica
}

\author{
$>$ Consolidation of Librarianship Postgraduate Studies in the University of Costa Rica
}

Ruth González-Arrieta ${ }^{1}$

\section{RESUMEN}

El artículo refleja la trayectoria del Programa de Posgrado en Bibliotecología de la Universidad de Costa Rica, que se implementó con el fin de formar un recurso humano especializado en la gestión de la información y los procesos documentales del país, y constituye una oferta académica única en la nación. A mediados de los 90, las Unidades Académicas de la Facultad de Educación conformaron una maestría académica que aglutinaba cursos con un tronco común. A su vez, cada escuela impartía cursos de su especialidad; en el caso de nuestra escuela, al posgrado se le denominó Maestría Académica en Educación con mención en Bibliotecología y Ciencias de la Información. Posteriormente, las Unidades Académicas de la Facultad, como la Escuela de Bibliotecología y Ciencias de la Información, ofertaron sus propias maestrías como el Programa de Posgrado en Bibliotecología y Estudios de la Información, que imparte la modalidad de la maestría profesional con énfasis en Gerencia de la Información y Tecnologías de la Información.

\section{ABSTRACT}

This article reflects the trajectory of the Postgraduate Program in Library Science from the University of Costa Rica, which was implemented in order to form specialized human resources in information management and document processes in the country. It constitutes a unique academic offering nationwide. In the mid 90's the Academic Units of the Faculty of Education, formed an academic master that brought together courses with a common core. In turn, each school taught courses in their own specialty; in the case of our school the master was named Academic Master in Education with a specialization in Library and Information Science. Subsequently, the Academic Units of the faculty, like the School of Librarianship and Information Science, tendered their own masters such as the Postgraduate Program in Librarianship and Information Studies, which teaches the modality of professional expertise with emphasis in Information Management and Information Technology.

Palabras clave

Maestría, profesionales, sociedad de la información, Costa Rica

\section{Keywords}

Master, professional, information society, Costa Rica

Recibido: 24 oct, 2014 | Corregido: 08 dic, 2014 | Aprobado: 09 dic, 2014

1 Universidad de Costa Rica, Escuela de Bibliotecología y Ciencias de la Información y Programa de Posgrado en Bibliotecología y Estudios de la Información. COSTA RICA. ruth.gonzalez@ucr.ac.cr 


\section{Introducción}

El mundo globalizado exige que los países formen personal con un perfil profesional altamente competitivo y especializado, con el fin de poder enfrentarse a los desafíos que les deparan los entornos nacional e internacional. Por ello, se requiere capacitar recursos humanos con las habilidades necesarias y una amplia visión para abordar estos retos, y brindar soluciones pertinentes.

Ante esta realidad y con el fin de preparar a profesionales en los diversos campos del saber humano, las instituciones de Educación Superior como la Universidad de Costa Rica (UCR) ofrecen un amplio abanico de ofertas de posgrado en las seis áreas académicas establecidas en el Estatuto Orgánico (2005): Artes y Letras, Ciencias Agroalimentarias, Ciencias Básicas, Ciencias de la Salud, Ciencias Sociales e Ingenierías.

Por ende, para coadyuvar a profesionales que asumen retos innovadores en el país, y así formar estudiantes con los conocimientos necesarios y las competencias profesionales para desarrollar aptitudes de capacidad crítica, iniciativa y creatividad en la planificación y ejecución de proyectos, se diseñaron los posgrados como el Posgrado de Bibliotecología, que describe su evolución en el presente artículo.

\section{Referentes}

Seguidamente, se exponen algunos antecedentes teóricos de la evolución del Posgrado de Bibliotecología en la Universidad de Costa Rica.

\subsection{Maestría Académica en Ciencias de la Educación con mención en Bibliotecología y Ciencias de la Información}

Durante los 90, la Facultad de Educación conformó la Comisión del Posgrado en Educación, con el fin de desarrollar un programa interdisciplinario de posgrado vinculante a las escuelas estipuladas en el Estatuto Orgánico (2005): Administración Educativa, Bibliotecología y Ciencias de la Información, Educación Física y Deportes, Formación Docente y Orientación, y Educación Especial. Además de conformar las cinco escuelas, se integró el Instituto de Investigaciones para el Mejoramiento de la Educación Costarricense, que en la actualidad se denomina Instituto de Investigación en Educación. Asimismo, el Sistema de Estudios de Posgrado (SEP) regulaba el programa que condujo al grado de Magister Scientiae en Educación.

En relación con la conceptualización de maestría académica, el convenio de universidades estatales inherente al Consejo Nacional de Rectores (2004) estipula: 
La maestría académica profundiza y actualiza conocimientos principalmente para investigación que genere más conocimiento, por lo que ésta se constituye en su núcleo generador. Su plan de estudios es más individualizado por estudiante, no necesariamente ha de estar centrado en cursos fijos y al menos $30 \%$ de la carga académica del estudiante ha de estar dedicada a actividades de investigación. Esta modalidad culmina con un trabajo de investigación o tesis de grado, que deben defenderse ante un tribunal. (p. 5)

El plan de estudios del programa abarcaba tres áreas de trabajo académico: a) Formación teórica, b) Formación teórica-metodológica, c) Actividades académico-profesionales (SEP, 1995), y se impartían cursos con un tronco común en educación y otros en la especialidad de la Unidad Académica base. De esta manera, el posgrado se identificaba con el nombre de Maestría Académica en Educación con menciones en las diferentes disciplinas.

En el seno de cada Unidad Académica se nombraba una comisión para determinar los cursos propios de la especialidad. De acuerdo con las Actas 2 y 3 del 10 de marzo y 26 de mayo de 1992, respectivamente, y el Acta 1-93 en la Escuela de Bibliotecología y Ciencias de la Información, para iniciar el procedimiento pertinente, se estableció la comisión integrada por los miembros Dr. Jorge González Fernández, M.Sc. Rodrigo Barrantes Echavarría, M.Ls. Luis Barrantes Rivera, Dra. Ana C. Torres, M.Ls. Benilda Salas Sánchez, Licda. Ana Lorena Echavarría Solís y M.Ls. Lidiette Díez Solano.

Posterior al proceso académico llevado a cabo por los miembros de la comisión, la Oficina de Planificación de Educación Superior adscrita al Consejo Nacional de Rectores, mediante el director M.Sc. José Andrés Masís Bermúdez (OPES-470-94-A), comunicó el 18 de octubre de 1994 al Dr. Luis Garita Bonilla, Rector de la Universidad de Costa Rica, que se avala la apertura de la mención solicitada. En el documento indicado, se aprobó tanto la apertura de una maestría académica como una profesional. Como se trataba de una estructura novedosa en la Facultad de Educación, la cual agrupaba varias Unidades Académicas que compartían cursos de un núcleo común, se inició con la maestría académica.

Por ende, la primera generación de la Maestría Académica en Educación con mención en Bibliotecología y Ciencias de la Información inició en el año lectivo de 1995 con los profesores Dr. Albam Brenes Chacón, M.Sc. Orlando Arboleda Sepúlveda, M.Sc. Rodrigo Barrantes Echavarría, Dra. Alicia Gurdián, M.Sc. Saray Córdoba González, M.Sc. Flor Cubero Venegas, Dra. Olimpia López Avendaño, Dr. Jacinto Ordónez Peñalonzo y el Dr. Walter Salazar Rojas.

La malla curricular abarcaba 60 créditos distribuidos en 30 créditos de cursos, 24 créditos de investigación y 6 créditos del trabajo final de graduación, detallados de la siguiente forma: Sociología del desarrollo de la educación, Enfoques metodológicos de la investigación socioeducativa, Taller de investigación 1, Análisis histórico político de la educación costarricense, Taller de investigación 2, La información y el desarrollo, Métodos de investigación cuantitativa, Taller de investigación 3, Gerencia de la información, Comercialización de la información, Evaluación de servicios de información, Taller de investigación 4 y Tesis o Trabajo de graduación. 
El grupo pionero de estudiantes estuvo integrado por Álvaro Pérez Guzmán, Zulay Calvo Abarca, María Elena Ureña Jiménez, Elizabeth Villalobos Sáenz, María Eugenia Briceño Meza, y Rosa M. Morales Rodríguez, y constituyó la primera promoción que realizó el plan de estudios mencionado con miras a obtener el título Magister Scientiae en Educación con énfasis en Bibliotecología y Ciencias de la Información.

Aunado a lo descrito, la graduanda M.Sc. Calvo, quien funge como docente de la Escuela de Bibliotecología y Ciencias de la Información, y es la directora de la biblioteca del Instituto sobre Alcoholismo y Farmacodependencia (IAFA) expresó que "los conocimientos adquiridos durante la maestría han sido de un incalculable valor académico para mi desarrollo profesional, así, como docente de la Unidad Académica" (Calvo Abarca, comunicación personal, 15 de octubre del 2014).

\section{Programa de Maestría en Bibliotecología y Estudios de la Información}

En el último lustro de los 90, en la Escuela de Bibliotecología y Ciencias de la Información se integró la comisión conformada por Rodrigo Barrantes Echavarría, Sonia Castillo Salas, Magda C. Sandí Sandí, Benilda Salas Sánchez y Ana C. Torres Muñoz, para diseñar una maestría propia de la disciplina, denominada Maestría en Bibliotecología y Estudios de la Información.

La nueva oferta académica se presentó ante el Sistema de Estudios de Posgrado, instancia que la elevó al Rector de la Universidad de Costa Rica, Dr. Fernando Durán Ayanegui, quien, a su vez, envió la propuesta académica (R-6950-99) el 23 de noviembre de 1999 al Consejo Nacional de Rectores (CONARE) para continuar los procedimientos establecidos en el fluxograma de la creación de nuevas carreras. Motivo por el cual el CONARE, como ente rector de las universidades públicas, durante la sesión 32-99 del 14 de diciembre de 1999, acordó que la Oficina de Planificación de la Educación Superior (OPES) realizara el estudio pertinente. Luego, la OPES plasmó la resolución en el documento OPES 4-2000.

Como lo establecía el dictamen de OPES, el Consejo Nacional de Rectores en la sesión 02-00, artículo 4, inciso j, del 15 de febrero del 2000, aprobó el Programa de la Maestría en Bibliotecología y Estudios de la Información, el cual comprendía una maestría académica y una profesional con dos énfasis: uno en Gerencia y otro en Tecnología de Computación, ambos adscritos al Sistema de Estudios de Posgrado.

\subsection{Maestría Profesional con énfasis en Gerencia de la Información}

Como el Consejo Nacional de Rectores avaló desarrollar ambos tipos de maestrías, en la Escuela de Bibliotecología y Ciencias de la Información se eligió impartir la maestría profesional.

Así, nuestra Unidad Académica cristalizó el objetivo propuesto con la apertura de la primera promoción de la en el año lectivo del 2000. 
En la nomenclatura para las universidades estatales establecida por el Consejo Nacional de Rectores (2004), se define este tipo de modalidad de la siguiente manera:

La maestría profesional profundiza y actualiza conocimiento, con el objeto primordial de analizarlo, sintetizarlo, transmitirlo y solucionar problemas...La investigación práctica aplicada se da a través de estudios de casos, diagnósticos y propuestas, producción artística y documental, laboratorios, prácticas profesionales, etc. Esta investigación debe evidenciarse en uno o varios informes y en una presentación final. (p. 6)

Asimismo, el Reglamento General del Sistema de Estudios de Posgrado (1995) estipula que cada posgrado debe constituir una comisión para el análisis académico y administrativo del posgrado. En el caso de Bibliotecología, la primera comisión de la maestría profesional la integraron los docentes de la Unidad Académica M.Sc. Rodrigo Barrantes Echavarría, Dra. Ana C. Torres Muñoz, MBA. Marcela Gil Calderón, Mag. Damaris Espinoza Quesada, Mag. Sonia Castillo Salas, MBA Magda C. Sandí Sandí y M.Ls. Benilda Salas Sánchez. Entre las funciones básicas de esta comisión se encuentra el análisis de los atestados de los postulantes a la maestría para determinar el cumplimiento de los requisitos de ingreso estipulados tanto por el SEP como por el propio programa.

La Unidad Académica determinó ejecutar y desarrollar el posgrado en la modalidad de financiamiento complementario, la cual se autofinancia, de acuerdo con la normativa universitaria, con el aporte de la matrícula de los estudiantes de cada ciclo lectivo.

En la oferta académica aprobada por el Consejo Nacional de Rectores para la maestría profesional, se establece una malla curricular de 18 cursos con una carga académica de 60 créditos; de estos, algunos corresponden al tronco común para ambos énfasis.

Los cursos definidos del núcleo común para los dos énfasis son: Pensamiento bibliotecológico, Paradigmas y enfoques de la investigación, Formulación y evaluación de proyectos, Tecnología para la administración de la información en bibliotecas, Competitividad y desarrollo empresarial, Taller de investigación práctico-aplicado 1 y Taller de investigación práctico-aplicado 2.

Por otro lado, los cursos de la especialidad en Gerencia de la Información son: Estudios bibliométricos, Información y sociedad, Aplicaciones métricas en la gestión de la información, Mercadeo de los servicios de la información, Procesos de liderazgo, Gerencia de la información, Análisis del entorno, Teoría organizacional, Comunicación y desarrollo empresarial, Legislación aplicada a las unidades de información y Psicología organizacional.

Paralelo a estos, debido a que se admiten estudiantes de otras disciplinas afines a Bibliotecología, se desarrolló el curso de nivelación Principios de bibliotecología.

Desde la primera promoción de la Maestría Profesional en Bibliotecología y Estudios de la Información con énfasis en Gerencia de la Información hasta el año 2010, han egresado cinco promociones, durante las cuales fungieron como directoras la Dra. Ana C. Torres Muñoz y la Mag. Ruth González Arrieta. 


\section{2.}

\section{Maestría Profesional con énfasis en Tecnologías de la Información}

En el 2009, en el marco de la sociedad de la información y la demanda de las tecnologías en los entornos nacional e internacional, la comisión del posgrado analizó la pertinencia de abrir la oferta académica para la Maestría Profesional con énfasis en Tecnología de la Computación, por lo cual inició los procedimientos académicos. Entre las deliberaciones de la comisión, se consideró que el nombre no era apropiado, ya que en la Universidad de Costa Rica se desarrollaba el posgrado de computación, por consiguiente, se propuso el nombre del énfasis como Tecnologías de la Información.

Asimismo, se actualizó la malla curricular con cursos innovadores a los cuales se les modificó tanto el nombre como los contenidos. Además de los cursos del tronco común de la disciplina bibliotecológica en la especialidad de Tecnologías de la Información, se impartieron los cursos Fundamentos de informática, Bases de datos, Diseño de bases de datos para unidades de información, Biblioteca virtual, Migración de datos, Práctica, Aplicaciones telemáticas en unidades de información, Análisis de sistemas y servicios de información basados en aplicaciones de internet para bibliotecas y Tecnología para la administración de la información en bibliotecas. Al curso Gerencia de la información se le cambió el nombre por el de Gestión de la información, y el de Información y sociedad se modificó por Sociedad de la información y del conocimiento.

En octubre del año 2011, la decana del Sistema de Estudios de Posgrado, la Dra. Gabriela Marín Raventós, comunicó mediante oficio SEP-1458-2011 la resolución del Consejo Nacional de Rectores con el aval de la apertura del énfasis en Tecnologías de la Información.

Por consiguiente, en el verano del 2012 inició la primera generación de la Maestría Profesional de Bibliotecología y Estudios de la Información con énfasis en Tecnologías de la Información.

En el actual año de 2014 profesores de la Escuela de Bibliotecología y Ciencias de la Información se desempeñan como integrantes de la comisión. Estos son: Ricardo Chinchilla Arley, Ruth González Arrieta, Xinia Rojas González, Zulay Calvo Abarca, Esteban González Pérez y Mynor Fernández Morales. Ellos velan por la calidad y excelencia académica del posgrado. Paralelo a la comisión, el posgrado ha logrado la estabilidad laboral de Jonathan Víquez Chacón, quien realiza las funciones administrativas y operativas acordes a la normativa y los sistemas implementados en la Universidad de Costa Rica.

Entre el cuerpo colegiado de docentes de ambos énfasis de la maestría fungen: Álvaro Pérez Guzmán, Damaris Espinoza Quirós, Zulay Calvo Abarca, Jacqueline García Fallas, Flora Jiménez Quesada, Isidora Chacón Álvarez, Ruth González Arrieta, Ana Beatriz Azofeifa Mora, Mauricio Víquez Lizano, Leda Abdala Arrieta, Orlando Arboleda Sepúlveda, Magda C. Sandí Sandí, Marcela Gil Calderón, Oscar Garbanzo Chinchilla, Esteban González Pérez, Cristóbal Pérez Jeréz, Ana C. Torres Muñoz, Saray Córdoba González, Rebeca Vargas Bolaños, Silvia Espinoza Tosi, María Ester Morales Ramírez, Gerardo Sánchez Ambriz, Aylin Valdivia Azze, Gustavo Sandí Calderón, Laura Rodríguez Amador, Sonia Grainger Burton, Ana C. Chávez, Egérico Porras, María Lourdes Flores de la Fuente, Lucía Jiménez Gómez, Max Cerdas López, 
Sonia Castillo Salas, Carmen Marín Baratta, Ricardo Chinchilla Arley, Jorge Quesada Araya, Hugo Cáceres, Anayancy Vallejos Quirós, Hernán Monterrosa Rojas, Silvia Hidalgo Sánchez, Mayra Ríos Hernández, Alexandra Arias Vindas, Lucía Jiménez Gómez, Verónica Chinchilla Fallas, Luis Loría Chavarría, Alonso Castro Mattey, Sonia Mora González y María del Carmen Sequeira Pérez, quienes transmiten el conocimiento a los futuros graduandos.

\section{Estudiantes del posgrado}

El Posgrado de Bibliotecología conserva los expedientes de los estudiantes desde el año 1996 hasta el 2014, que registran los atestados que analiza la comisión en el proceso de admisión y otros documentos de la vida académica del estudiante. Esta información refleja que el $95 \%$ de los estudiantes del posgrado provienen del grado de licenciatura de la carrera de Bibliotecología. No obstante, la comisión admite educandos con el grado de bachillerato universitario, como lo establece la normativa de la Universidad de Costa Rica. Asimismo, se aceptan estudiantes de otras carreras que desarrollan su profesión en el ámbito de las unidades de información como archivistas, administradores de empresas, filólogos, estadistas, orientadores y educadores, entre otros. Esto implica un abanico de pensamientos interdisciplinarios que enriquece la profesionalidad del posgrado. Aunado a los registros de los expedientes estudiantiles, en general, se trata de adultos jóvenes con experiencia laboral, cualidades que la comisión valora en la selección de los educandos, ya que les permite conllevar el reto y esfuerzo que requiere la maestría para obtener el rendimiento académico deseado y alcanzar los frutos propuestos como profesionales.

En ambos énfasis, paralelo a los cursos de investigación, los estudiantes elaboran el Trabajo Final de Investigación Aplicada (TFIA) en la modalidad de Práctica Dirigida o Proyecto de Graduación, guiados por un Comité Asesor integrado por el tutor y dos lectores, los cuales orientan al estudiante en el TFIA para realizar la defensa pública frente a un tribunal examinador y así culminar con el título de maestría o magister. Además de esta defensa, los estudiantes presentan su investigación a la comunidad nacional durante las Jornadas de Investigación Bibliotecológica, organizadas por el posgrado en forma bianual.

La mayoría de los estudiantes ejecutan el trabajo final en sus lugares de trabajo con la venia de los empleadores, debido a que se trata de un trabajo riguroso e innovador, e induce a cambios sustanciales en la institución donde se realice, como se detalla a continuación (Ver tabla 1, 2 y 3): 


\section{Tabla 1}

Lista de tesis de los egresados de la Maestría Académica en Educación con mención en Bibliotecología y Ciencias de la Información hasta el 2014

\begin{tabular}{|c|c|}
\hline Estudiantes & Tesis \\
\hline Rosa María Morales Rodríguez & $\begin{array}{l}\text { Estudio sobre las necesidades de información del profesional } \\
\text { en ingeniería del Ministerio de Obras Públicas y Transportes. }\end{array}$ \\
\hline Zulay Calvo Abarca & $\begin{array}{l}\text { Perfil académico profesional del Bachiller en Bibliotecología de } \\
\text { la Universidad de Costa Rica. }\end{array}$ \\
\hline Elizabeth Villalobos Sáenz & El bibliotecólogo como gerente de la información \\
\hline Álvaro Guzmán Pérez & $\begin{array}{l}\text { Las necesidades de información de las Instituciones Teológicas } \\
\text { Protestantes Latinoamericanas (desde la perspectiva } \\
\text { académica). Propuesta de Servicios y Productos de } \\
\text { Información }\end{array}$ \\
\hline
\end{tabular}

Fuente: Posgrado de Bibliotecología, 2014.

Tabla 2

Lista de tesis de los egresados de la Maestría Profesional en Bibliotecología y Estudios de la Información con énfasis en Gerencia de la Información hasta el 2014

\begin{tabular}{|c|c|}
\hline Estudiantes & Trabajo Final de Investigación Aplicada \\
\hline Yamilette Solano Navarro & $\begin{array}{l}\text { Repertorio de las Revistas de Costa Rica publicadas en } \\
\text { el periodo de } 1850 \text { a } 1899 \text { y de } 1900 \text { a } 1959 \\
\text { pertenecientes a la Biblioteca Nacional "Miguel Obregón } \\
\text { Lizano" }\end{array}$ \\
\hline Erick Montoya Trejos & $\begin{array}{l}\text { Propuesta de contenidos de una página web con } \\
\text { información del proceso de admisión e ingreso a la } \\
\text { Universidad de Costa Rica. }\end{array}$ \\
\hline Katia Moraga Vega & $\begin{array}{l}\text { Pertinencia de los recursos de información de la } \\
\text { Biblioteca Martin Lees: análisis bibliométrico en tesis de } \\
\text { Maestría en Derecho Internacional y Derechos Humanos } \\
\text { de la Universidad de la Paz }\end{array}$ \\
\hline Esteban González Pérez & $\begin{array}{l}\text { Estrategias didácticas desde la perspectiva } \\
\text { constructivista apoyadas con medios digitales para la } \\
\text { Escuela de Bibliotecología y Ciencias de la Información, } \\
\text { Universidad de Costa Rica }\end{array}$ \\
\hline $\begin{array}{l}\text { Tayira Hernández Guzmán } \\
\text { Indira Bermúdez Aguilar }\end{array}$ & $\begin{array}{l}\text { La conceptualización de la creación del portal de } \\
\text { servicios en el Instituto de Investigación en Educación de } \\
\text { la Universidad de Costa Rica }\end{array}$ \\
\hline Cristina Rocha Palma & $\begin{array}{l}\text { Estudio bibliométrico de la producción científica de la } \\
\text { Revista Reflexiones de la Facultad de Ciencias Sociales } \\
\text { de la Universidad de Costa Rica: Periodo 1992-2011 }\end{array}$ \\
\hline $\begin{array}{l}\text { Aura Sánchez Velásquez } \\
\text { Irina Garrido Cordero }\end{array}$ & $\begin{array}{l}\text { Alfabetización informacional para los usuarios de la } \\
\text { Biblioteca Joaquín García Monge del Sistema de } \\
\text { Información Documental de la Universidad Nacional } \\
\text { (SIDUNA) }\end{array}$ \\
\hline
\end{tabular}


Ana María Leiva González Florangel Catálogo digital para optimizar la información de las Rodríguez Ramírez Verónica Chinchilla Fallas

bases de datos, revistas suscritas y académicas producidas por la Universidad de Costa Rica para los docentes en régimen académico de las áreas agroalimentarias, ingeniería y salud de la Sede Rodrigo Facio

Sofía Castillo Ruiz

La motivación laboral de los y las profesionales de archivística y bibliotecología de la Universidad de Costa Rica.

Elieth Angulo Varela

Modelo de gestión de información de la Biblioteca José Figueres Ferrer para los investigadores e investigadoras del Instituto Tecnológico de Costa Rica.

Patricia Sánchez López Gestión de colecciones: evaluación de los libros de la Colección General de la Biblioteca Carlos Monge Alfaro de la Universidad de Costa Rica

Elvia Ramírez Ramírez

Las poetas centroamericanas en el Repertorio Americano de 1919 a 1959: patrimonio documental digital.

Rocío Pérez Brenes Indicadores de desempeño ISO 11620 y UNE 50137 para la gestión bibliotecológica en las Bibliotecas Médicas de Hospitales Desconcentrados de la Provincia de San José.

Alexandra Arias Vindas

Silvia Elena Jerez Brenes

María del Rocío Sáenz Quesada

Daniel Zúñiga Van der Laat

Agr

Pedro Montero Bustabad

José Pablo Meza Pérez

Yetty Chavarría Alemán

Fressy Ramírez Esquivel

María Elena Céspedes Rodríguez
Modelo digital para la compra de recursos de información bibliográfica en el Sistema de Bibliotecas, Documentación e Información (SIBDI) de la Universidad de Costa Rica.

Estrategia para optimizar la gestión de la información técnica agropecuaria generada por el Ministerio de Agricultura y Ganadería.

Portal teológico para América Latina

Operacionalización de una red virtual de conocimiento para los Productores Documentales e Investigadores en Administración Pública y Finanzas para Centroamérica, Panamá y República Dominicana

Propuesta para la gestión normalizada de las publicaciones institucionales del ICE

Manual de conservación y preservación de los libros impresos en la Biblioteca Monseñor Sanabria Martínez de la Asamblea Legislativa

La gestión de la información por medio del Cuadro de Mando Integral en el Centro Integrado de Documentación Centroamericana en Ciencias Sociales (CIDACS) de la Universidad de Costa Rica 
Continuación de la tabla $2 .$.

\begin{tabular}{|c|c|}
\hline Estudiantes & Trabajo Final de Investigación Aplicada \\
\hline María Carlina Rojas Badilla & $\begin{array}{l}\text { Propuesta de una Intranet para el Sistema de } \\
\text { Información Documental de la Universidad Nacional } \\
\text { (SIDUNA) }\end{array}$ \\
\hline Ma. Bernardita Miranda Barquero & $\begin{array}{l}\text { Propuesta para implantar servicios virtuales de } \\
\text { información con soporte web en el Centro de Información } \\
\text { en el Banco de Costa Rica }\end{array}$ \\
\hline Monserrath Corrales Blanco & $\begin{array}{l}\text { Propuesta para crear la Biblioteca Virtual del Cantón de } \\
\text { Moravia. }\end{array}$ \\
\hline Leda María Arguedas Ferreto & $\begin{array}{l}\text { Estudio de usuarios en la Biblioteca Eugenio Fonseca } \\
\text { Tortós: propuesta de un modelo de estudios métricos } \\
\text { para la toma de decisiones }\end{array}$ \\
\hline Evelyn Barrientos Villalta & $\begin{array}{l}\text { Propuesta de creación de la Biblioteca Digital del } \\
\text { Consejo Nacional de Rectores }\end{array}$ \\
\hline Ligia Patricia Montero Valverde & $\begin{array}{l}\text { Propuesta de un modelo de catalogación cooperativa } \\
\text { para el Sistema Bibliotecario de la Educación Superior } \\
\text { Universitaria Estatal de Costa Rica (SIBESE-CR) }\end{array}$ \\
\hline Ana Lorena Sánchez Ulate & $\begin{array}{l}\text { Propuesta para implementar el pago de servicios } \\
\text { bibliográficos a través de Internet para la Unidad de } \\
\text { Información del Centro Nacional de Ciencia y Tecnología } \\
\text { de Alimentos (CITA) de la Universidad de Costa Rica }\end{array}$ \\
\hline Marjorie Solano Siles & $\begin{array}{l}\text { Propuesta de un plan piloto para la alfabetización } \\
\text { informacional en la Facultad de Ciencias Sociales de la } \\
\text { Universidad de Costa Rica }\end{array}$ \\
\hline Paola Barquero Sanabria & $\begin{array}{l}\text { Uso de indicadores de rendimiento en el Centro } \\
\text { Catalográfico del Sistema de Bibliotecas, Documentación } \\
\text { e Información (SIBDI) de la Universidad de Costa Rica: } \\
\text { una propuesta }\end{array}$ \\
\hline $\mathrm{M}^{\mathrm{a}}$ Lourdes Flores de la Fuente & $\begin{array}{l}\text { Sistema de gestión de calidad para el Centro de } \\
\text { Documentación de ILANUD según la Norma ISO } \\
9001: 2000\end{array}$ \\
\hline Flor María Soto Arroyo & $\begin{array}{l}\text { Propuesta para la implementación de tecnología de } \\
\text { información y comunicación en los Servicios de } \\
\text { Referencia de la Biblioteca Arturo Agüero Chaves de la } \\
\text { Sede Regional de Occidente de la Universidad de Costa } \\
\text { Rica }\end{array}$ \\
\hline $\begin{array}{l}\text { Ana Julia Arguedas Valverde } \\
\text { Floribeth Sánchez Espinoza }\end{array}$ & $\begin{array}{l}\text { Propuesta de creación de una unidad de información en } \\
\text { Ecoturismo }\end{array}$ \\
\hline Margot Azofeifa Castro & $\begin{array}{l}\text { Biblioteca comunitaria en el Distrito de Guaitil del cantón } \\
\text { de Acosta: una propuesta para su implementación }\end{array}$ \\
\hline Ana Beatriz Azofeifa Mora & $\begin{array}{l}\text { Proyecto "BINABITROP-América Central", una } \\
\text { herramienta para la gestión de la información ambiental }\end{array}$ \\
\hline María Teresa Bermúdez M. & $\begin{array}{l}\text { El impacto del expediente médico en la gestión } \\
\text { hospitalaria. Estudio de caso: el archivo clínico del } \\
\text { Hospital Dr. Rafael Ángel Calderón Guardia }\end{array}$ \\
\hline
\end{tabular}


Continuación de la tabla $2 . .$.

\begin{tabular}{|c|c|}
\hline Estudiantes & Trabajo Final de Investigación Aplicada \\
\hline Nuria Calderón Marín & $\begin{array}{l}\text { Propuesta de creación de un sistema institucional de } \\
\text { gestión de información y documentación en la } \\
\text { Municipalidad de San José }\end{array}$ \\
\hline $\begin{array}{l}\text { Olga Isabel Castro Gutiérrez } \\
\text { María Luisa Chavarría Jiménez }\end{array}$ & $\begin{array}{l}\text { El aporte del género femenino en la historia de la } \\
\text { Asamblea Legislativa de la República de Costa Rica de } \\
1990 \text { al } 2004\end{array}$ \\
\hline Rosa Eugenia Hernández Briceño & $\begin{array}{l}\text { Propuesta para la creación de un servicio de información } \\
\text { para pacientes en el Hospital Dr. Rafael Ángel Calderón } \\
\text { Guardia. }\end{array}$ \\
\hline Doris Lucia Jiménez Gómez & $\begin{array}{l}\text { La inteligencia emocional: un recurso efectivo en la } \\
\text { prestación de servicios de calidad en las bibliotecas } \\
\text { universitarias }\end{array}$ \\
\hline Rosa Murguía Paiz & $\begin{array}{l}\text { Gestión de calidad en los servicios del Centro de } \\
\text { Documentación e Información del INII de la Facultad de } \\
\text { Ingeniería }\end{array}$ \\
\hline $\begin{array}{l}\text { Silvia Navas Orozco } \\
\text { Damaris Rodríguez González }\end{array}$ & $\begin{array}{l}\text { Propuesta para implementar un servicio de biblioterapia } \\
\text { en la Biblioteca de Pacientes del Hospital Nacional } \\
\text { Psiquiátrico. }\end{array}$ \\
\hline Guillermo Pérez Merayo & $\begin{array}{l}\text { Centro para la investigación y la enseñanza del gobierno } \\
\text { electrónico y de la economía digital: un sitio web }\end{array}$ \\
\hline Isabel María Tan Chan & $\begin{array}{l}\text { Aporte de las bibliotecas públicas en la sociedad } \\
\text { costarricense de la información }\end{array}$ \\
\hline Joaquín Alfredo Víquez Abarca & $\begin{array}{l}\text { Aplicación del principio de procedencia en la gestión de } \\
\text { documentos electrónicos }\end{array}$ \\
\hline Nuria María Zamora Chavarría & $\begin{array}{l}\text { Plan de desarrollo estratégico para los servicios de } \\
\text { información de la Sede Regional Chorotega y el Centro } \\
\text { de Investigación de Desarrollo Sostenible del Trópico } \\
\text { Seco (CEMEDE) de la Universidad Nacional en Nicoya }\end{array}$ \\
\hline $\begin{array}{l}\text { Mónica Arce Oviedo } \\
\text { Lidilia Carballo Arguedas } \\
\text { Carlos Quirós Álvarez }\end{array}$ & $\begin{array}{l}\text { Necesidades de información de la población infantil de la } \\
\text { Escuela del Barrio El Carmen de Puntarenas para el } \\
\text { proyecto de biblioteca infantil ecológica del parque } \\
\text { Marino del Pacífico: un estudio de caso }\end{array}$ \\
\hline $\begin{array}{l}\text { Idahyma Barrantes Fernández } \\
\text { Amalia Bell Manzanares }\end{array}$ & $\begin{array}{l}\text { La red Internet como herramienta de apoyo para la } \\
\text { gestión y uso de la información sobre la mujer en la } \\
\text { colectiva feminista Francisca Carrasco }\end{array}$ \\
\hline $\begin{array}{l}\text { Patricia Castro Araya } \\
\text { Marjorie Pérez Chavarría }\end{array}$ & $\begin{array}{l}\text { Incidencia de la cultura organizacional en el desempeño } \\
\text { de la Biblioteca Carmen María Chaverri del Liceo } \\
\text { Napoleón Quesada Salazar }\end{array}$ \\
\hline William Cortés Ramírez & $\begin{array}{l}\text { Restauración de documentos textuales en soporte de } \\
\text { papel en Costa Rica }\end{array}$ \\
\hline Flory Gamboa Umaña & $\begin{array}{l}\text { Impacto del uso de las bases de datos del SIBDI por los } \\
\text { investigadores del área de Ciencias Sociales de la } \\
\text { Universidad de Costa Rica }\end{array}$ \\
\hline Laura María Guevara Rojas & $\begin{array}{l}\text { Gerencia de la información en los Trabajos Finales de } \\
\text { Graduación de la Escuela de Bibliotecología de la UCR y } \\
\text { en la Revista AIBDA. }\end{array}$ \\
\hline
\end{tabular}


:Continuación de la tabla $2 . .$.

\begin{tabular}{|l|l|}
\hline \multicolumn{1}{|c|}{ Estudiantes } & \multicolumn{1}{|c|}{ Trabajo Final de Investigación Aplicada } \\
\hline Mónica del Rosario Isaza Zapata & $\begin{array}{l}\text { Gestión documental según ISO/DIS 17025 a través de la } \\
\text { Intranet en el Laboratorio Control de Calidad el Alto, } \\
\text { RECOPE }\end{array}$ \\
\hline $\begin{array}{l}\text { Rosa León Valverde } \\
\text { Lupita Rodríguez Méndez }\end{array}$ & $\begin{array}{l}\text { Estudio de viabilidad para la transformación del centro } \\
\text { de información y documentación OTUS del Instituto } \\
\text { Nacional de Biodiversidad en un centro de información } \\
\text { digital }\end{array}$ \\
\hline Maribel Vallejos Vásquez & $\begin{array}{l}\text { La digitalización como soporte para la gestión } \\
\text { documental: el caso del sistema de gestión documental } \\
\text { del Instituto Costarricense de Electricidad }\end{array}$ \\
\hline Rebeca Vargas Bolaños & $\begin{array}{l}\text { Aporte científico: una década de investigación en el } \\
\text { IIMEC. }\end{array}$ \\
\hline
\end{tabular}

Fuente: Posgrado de Bibliotecología, 2014.

\section{Tabla 3}

Lista de tesis de los egresados de la Maestría Profesional en Bibliotecología y Estudios de la Información con énfasis en Tecnologías de la Información hasta el 2014

\begin{tabular}{|l|l|}
\hline \multicolumn{1}{|c|}{ Estudiante } & \multicolumn{1}{c|}{ Trabajo Final de Investigación Aplicada } \\
\hline Loirette Calvo Sánchez & $\begin{array}{l}\text { Utilización de herramientas colaborativas para el diseño de una guía } \\
\text { didáctica digital para los cursos de procesos técnicos de la Escuela } \\
\text { de Bibliotecología y Ciencias de la Información de la Universidad de } \\
\text { Costa Rica. }\end{array}$ \\
\hline
\end{tabular}

Fuente: Posgrado de Bibliotecología, 2014

\section{Conclusiones}

Desde los inicios del Posgrado en Bibliotecología, los estudiantes han sido altamente competitivos en las proyecciones académicas y profesionales, lo que les ha permitido culminar su vida académica con el trabajo final de graduación.

Se determina que diversas instituciones de la sociedad costarricense, se han enriquecido con los trabajos de los graduandos del Posgrado en Bibliotecología, ya que la mayoría los ejecutan en los lugares de trabajo del educando, solucionando problemas en la gestión de la información de la entidad.

El Posgrado en Bibliotecología ha contribuido en la formación de profesores de la Unidad Académica, ya que al desempeñarse como docentes, tienen la experticia profesional en la actualización e innovación de conocimientos que incide al formar los estudiantes. 
Con el transcurso del tiempo, se evidencia en la presente investigación, la evolución del Programa del Posgrado en Bibliotecología de la Universidad de Costa Rica, y a su vez, la consolidación visionaria de hombres y mujeres que han legado sus conocimientos, en la formación de profesionales comprometidos por el desarrollo del país.

\section{Bibliografía}

Consejo Nacional de Rectores. (2000). Dictamen sobre la propuesta de creación de la Maestría en Bibliotecología y Estudios de la Información en la Universidad de Costa Rica. OPES-4-2000. San José, Costa Rica: CONARE, OPES.

Consejo Nacional de Rectores. (2004). Leyes, Convenios y Decretos de la Educación Superior Universitaria Estatal en Costa Rica (3a ed.). San José, Costa Rica: CONARE, OPES.

Consejo Universitario de la Universidad de Costa Rica. (2005). Estatuto Orgánico (8a ed.). San José: SIEDIN.

Escuela de Bibliotecología y Ciencias de la Información. (2009). Posgrado en Bibliotecología [sitio web]. Recuperado de http://www.ebci.ucr.ac.cr/posgrado

Maestría en Bibliotecología y Estudios de la Información. (2008a). Maestría Profesional en Bibliotecología énfasis en Gerencia de la Información. CAPE. San José, Costa Rica: Autor.

Maestría en Bibliotecología y Estudios de la Información. (2009b). Programa de Posgrado de la Maestría Profesional en Bibliotecología énfasis en Gerencia de la Información. SEP. San José, Costa Rica: Autor.

Sistema de Estudios de Posgrado. (1995). Maestría en Ciencias de la Educación. Programa de Estudios. San José, Costa Rica: Comisión del Posgrado en Educación.

Sistema de Estudios de Posgrado. (1995). Reglamento General del Sistema de Estudios de Posgrado. Recuperado de http://www.cu.ucr.ac.cr/normativ/sistema_estudios_posgrado.pdf 


\section{Sección Especial del 25 Aniversario de la EBCI}

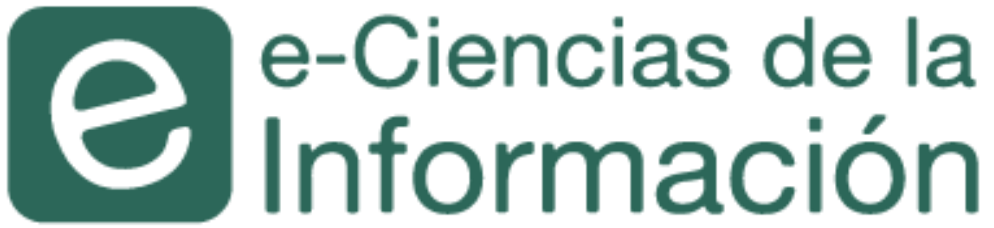

¿Desea publicar su trabajo? Ingrese aquí

O escríbanos a la siguiente dirección: revista.ebci@ucr.ac.cr

(우(1)(2)

\section{1}

Origen: respuesta a una necesidad

\section{En el año 2011, la Escuela de}

Bibliotecología y Ciencias de la Información (EBCl) de la Universidad de Costa Rica (UCR) reconoció la importancia de crear nuevas y mejores alternativas para difusión de la investigación. e-Ciencias de la Información es la respuesta a un contexto actual marcado por una mayor apertura, flexibilidad, y rigurosidad en la verificación de los datos y su procesamiento.

\section{3}

Primer cuartil-Revistas UCR

e-Ciencias de la Información es una revista científica que aborda las nuevas temáticas de desarrollo e investigación en las Ciencias de la Información, en el ámbito nacional e internacional. Así, colabora significativamente en el progreso de esta disciplina. Por sus parámetros de calidad, pertenece al primer cuartil de la UCR y se encuentra ampliamente indizada en los importantes catálogos.

\section{En la actualidad}

\section{Posicionamiento internacional}

La revista admite trabajos en las siguientes áreas, siempre que se relacionen directamente con las Ciencias de la Información:

Bibliotecología, Documentación, Tecnologías de la Información y la Comunicación, Investigación, Análisis Estadísticos y Bibliometría, Archivística, Sistemas de Información, Informática, Comunicación Colectiva y Bibliotecas Escolares.
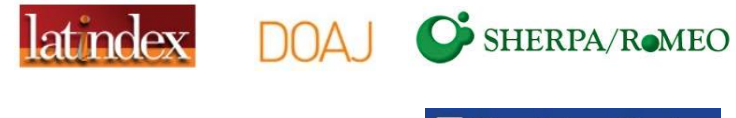

- Dialnet

e-revist@s

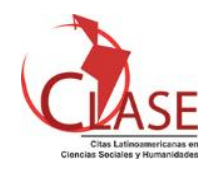

Google

e-prints in library \& 